\title{
Degenerate Bell polynomials associated with umbral calculus
}

\author{
Taekyun Kim', Dae San Kim², Han-Young Kim , Hyunseok Lee² and Lee-Chae Jang ${ }^{3 *}$
}

"Correspondence:

lcjang@konkuk.ac.kr

${ }^{3}$ Graduate School of Education, Konkuk University, Seoul 143-701, Republic of Korea

Full list of author information is

available at the end of the article

\section{是 Springer}

\begin{abstract}
Carlitz initiated a study of degenerate Bernoulli and Euler numbers and polynomials which is the pioneering work on degenerate versions of special numbers and polynomials. In recent years, studying degenerate versions regained lively interest of some mathematicians. The purpose of this paper is to study degenerate Bell polynomials by using umbral calculus and generating functions. We derive several properties of the degenerate Bell polynomials including recurrence relations, Dobinski-type formula, and derivatives. In addition, we represent various known families of polynomials such as Euler polynomials, modified degenerate poly-Bernoulli polynomials, degenerate Bernoulli polynomials of the second kind, and falling factorials in terms of degenerate Bell polynomials and vice versa.

MSC: 05A19; 05A40; 11B68; 11B73; 11B83

Keywords: Degenerate Bell polynomials; Umbral calculus; Euler polynomials; Degenerate Bernoulli polynomials of the second kind; Degenerate poly-Bernoulli polynomials
\end{abstract}

\section{Introduction and preliminaries}

In [3, 4], Carlitz studied degenerate Bernoulli and Euler polynomials, which are degenerate versions of the ordinary Bernoulli and Euler polynomials, and investigated some combinatorial results as well as some arithmetical ones. We have witnessed in recent years that, along the same line as Carlitz's pioneering work, some mathematicians began to explore degenerate versions of quite a few special polynomials and numbers which include the degenerate Bernoulli numbers of the second kind, degenerate Stirling numbers of the first and second kinds, degenerate Cauchy numbers, degenerate Bell numbers and polynomials, degenerate complete Bell polynomials and numbers, degenerate gamma function, and so on (see $[8,9,11,14-16,20]$ and the references therein). They have been studied by various means like combinatorial methods, generating functions, differential equations, umbral calculus techniques, $p$-adic analysis, and probability theory. Here we would like to point out that the generating functions are also useful in related fields like mathematical physics (see [23, 24]). Moreover, those degenerate versions of some special polynomials found some applications to other areas of mathematics such as differential equations, identities of symmetry, and probability theory $[12,13,18]$.

(c) The Author(s) 2020. This article is licensed under a Creative Commons Attribution 4.0 International License, which permits use sharing, adaptation, distribution and reproduction in any medium or format, as long as you give appropriate credit to the original author(s) and the source, provide a link to the Creative Commons licence, and indicate if changes were made. The images or other third party material in this article are included in the article's Creative Commons licence, unless indicated otherwise in a credit line to the material. If material is not included in the article's Creative Commons licence and your intended use is not permitted by statutory regulation or exceeds the permitted use, you will need to obtain permission directly from the copyright holder. To view a copy of this licence, visit http://creativecommons.org/licenses/by/4.0/. 
The Bell number $B_{n}$ counts the number of partitions of a set with $n$ elements into disjoint nonempty subsets. The Bell polynomials are natural extensions of Bell numbers and also called Touchard or exponential polynomials (see [2]). As a degenerate version of these Bell polynomials and numbers, the degenerate Bell polynomials and numbers in (18) are introduced and studied under the different names of the partially degenerate Bell polynomials and numbers in [16].

The purpose of the present paper is to study the degenerate Bell polynomials and numbers by means of umbral calculus and generating functions. We investigate several properties of those numbers and polynomials which include Dobinski-type formula, recurrence relations, and their derivatives. Moreover, with the help of umbral calculus techniques we represent various known families of polynomials such as Euler polynomials, modified degenerate poly-Bernoulli polynomials, degenerate Bernoulli polynomials of the second kind, and falling factorials in terms of degenerate Bell polynomials and vice versa. Even though the degenerate Bell polynomials were introduced earlier in [16], only some basic properties were studied there by using generating functions. The novelty of this paper is that they are further explored by employing a different method, namely umbral calculus. In particular, this enables us to represent various known families of polynomials in terms of degenerate Bell polynomials and vice versa, which can be viewed as a classical connection problem.

This paper is outlined as follows. In Sect. 1, firstly we recall the degenerate exponential functions, the degenerate logarithms, and the degenerate polylogarithm functions which are respectively degenerate versions of exponential functions, logarithms, and polylogarithm functions. Then we go over the definitions of some degenerate special polynomials and numbers, namely the modified degenerate poly-Bernoulli polynomials, the degenerate Bernoulli polynomials of the second kind of order $r$, the degenerate Stirling numbers of the first and second kinds, and the degenerate Bell polynomials. In addition, we briefly state some basic facts about umbral calculus. For details, we let the reader refer to [21]. In Sect. 2, firstly we derive Dobinski-type formulas, recurrence relations, and the derivatives for the degenerate Bell polynomials. Then we find a formula expressing any polynomial in terms of the degenerate Bell polynomials. We apply this formula to Euler polynomials and to find the inversion formula of (28). By applying the transfer formula we obtain a relation involving the degenerate Bell polynomials, Stirling numbers of the first and second kinds, and the higher-order degenerate Bernoulli polynomials of the second kind. In addition, by using the general formula (26) expressing one Sheffer polynomial in terms of another Sheffer polynomial, we express Euler polynomials and modified degenerate poly-Bernoulli polynomials in terms of the degenerate Bell polynomials and also represent the degenerate Bell polynomials in terms of falling factorials and degenerate Bernoulli polynomials of the second kind. Finally, Sect. 3 is the conclusion of this paper.

For any $0 \neq \lambda \in \mathbb{R}$, we recall that the degenerate exponential functions are defined by

$$
e_{\lambda}^{x}(t)=(1+\lambda t)^{\frac{x}{\lambda}}, \quad e_{\lambda}(t)=e_{\lambda}^{1}(t), \quad(\text { see }[9,14]) .
$$

Note that

$$
e_{\lambda}^{x}(t)=\sum_{n=0}^{\infty}(x)_{n, \lambda} \frac{t^{n}}{n !} \quad(\text { see }[9])
$$


where $\left.(x)_{0, \lambda}=1,(x)_{n, \lambda}=x(x-\lambda) \cdots(x-(n-1) \lambda)\right),(n \geq 1)$.

The degenerate logarithm $\log _{\lambda}(1+t)$, which is the compositional inverse of the degenerate exponential function $e_{\lambda}(t)$ and a motivation for the definition of degenerate polylogarithm function, is defined by

$$
\log _{\lambda}(1+t)=\sum_{n=1}^{\infty} \lambda^{n-1}(1)_{n, 1 / \lambda} \frac{t^{n}}{n !}=\sum_{n=1}^{\infty}(\lambda-1)_{n-1} \frac{t^{n}}{n !}=\frac{1}{\lambda}\left((1+t)^{\lambda}-1\right), \quad \text { (see [9]). }
$$

Note that $\lim _{\lambda \rightarrow 0} \log _{\lambda}(1+t)=\log (1+t), \log _{\lambda}\left(e_{\lambda}(t)\right)=e_{\lambda}\left(\log _{\lambda}(t)\right)=t$.

We recall from equation (12) of [9] that, for $k \in \mathbb{Z}$, the degenerate polylogarithm functions are defined by

$$
\operatorname{Li}_{k, \lambda}(x)=\sum_{n=1}^{\infty} \frac{(-\lambda)^{n-1}(1)_{n, 1 / \lambda}}{(n-1) ! n^{k}} x^{n} \quad(|x|<1)
$$

Note that

$$
\lim _{\lambda \rightarrow 0} \operatorname{Li}_{k, \lambda}(x)=\sum_{n=1}^{\infty} \frac{x^{n}}{n^{k}}=\operatorname{Li}_{k}(x)
$$

where $\mathrm{Li}_{k}(x)$ is called the polylogarithm.

From (4), we note that $\operatorname{Li}_{1, \lambda}(x)=-\log _{\lambda}(1-x)$. In [3, 4], Carlitz considered the degenerate Bernoulli polynomials which are given by

$$
\frac{t}{e_{\lambda}(t)-1} e_{\lambda}^{x}(t)=\sum_{n=0}^{\infty} \beta_{n, \lambda}(x) \frac{t^{n}}{n !}
$$

For $x=0, \beta_{n, \lambda}=\beta_{n, \lambda}(0)$ are called the degenerate Bernoulli numbers.

It is easy to show that $\lim _{\lambda \rightarrow 0} \beta_{n, \lambda}(x)=B_{n}(x),(n \geq 0)$, where $B_{n}(x)$ are the ordinary Bernoulli polynomials given by

$$
\frac{t}{e^{t}-1} e^{x t}=\sum_{n=0}^{\infty} B_{n}(x) \frac{t^{n}}{n !} \quad(\text { see }[1-18,20-22,25,26]) .
$$

For $x=0, B_{n}=B_{n}(0)$ are called the Bernoulli numbers.

It is well known that the Euler polynomials are given by

$$
\frac{2}{e^{t}+1} e^{x t}=\sum_{n=0}^{\infty} E_{n}(x) \frac{t^{n}}{n !} \quad(\text { see }[21]) .
$$

For $x=0, E_{n}=E_{n}(0)$ are called the Euler numbers.

From (7), we note that

$$
E_{n}(x)=\sum_{l=0}^{n}\left(\begin{array}{l}
n \\
l
\end{array}\right) E_{n-l} x^{l} \quad(n \geq 0)
$$


Recently, the degenerate poly-Bernoulli polynomials have been defined by means of the degenerate polylogarithms as follows:

$$
\frac{\operatorname{Li}_{k, \lambda}\left(1-e_{\lambda}(-t)\right)}{1-e_{\lambda}(-t)} e_{\lambda}^{x}(-t)=\sum_{n=0}^{\infty} B_{n, \lambda}^{(k)}(x) \frac{t^{n}}{n !} \quad(\text { see [9] }) .
$$

When $k=1$, we note that $B_{n, \lambda}^{(1)}(x)=(-1)^{n} \beta_{n, \lambda}(x),(n \geq 0)$. Now, we slightly modify the definition of the degenerate poly-Bernoulli polynomials, called the modified degenerate polyBernoulli polynomials, as follows:

$$
\frac{\operatorname{Li}_{k, \lambda}\left(1-e_{\lambda}(-t)\right)}{e_{\lambda}(t)-1} e_{\lambda}^{x}(t)=\sum_{n=0}^{\infty} \beta_{n, \lambda}^{(k)}(x) \frac{t^{n}}{n !} \quad(k \in \mathbb{Z}), \text { (see [17]). }
$$

Note that $\beta_{n, \lambda}^{(1)}(x)=\beta_{n, \lambda}(x),(n \geq 0)$. When $x=0, \beta_{n, \lambda}^{(k)}=\beta_{n, \lambda}^{(k)}(0)$ are called the modified degenerate poly-Bernoulli numbers.

The degenerate Bernoulli polynomials of the second kind of order $r$ are defined by

$$
\left(\frac{t}{\log _{\lambda}(1+t)}\right)^{r}(1+t)^{x}=\sum_{n=0}^{\infty} b_{n, \lambda}^{(r)}(x) \frac{t^{n}}{n !}
$$

For $x=0, b_{n, \lambda}^{(r)}=b_{n, \lambda}^{(r)}(0)$ are called the degenerate Bernoulli numbers of the second kind of order $r$. Note that

$$
\lim _{\lambda \rightarrow 0}\left(\frac{t}{\log _{\lambda}(1+t)}\right)^{r}(1+t)^{x}=\left(\frac{t}{\log (1+t)}\right)^{r}(1+t)^{x}=\sum_{n=0}^{\infty} b_{n}^{(r)}(x) \frac{t^{n}}{n !},
$$

where $b_{n}^{(r)}(x)$ are called the Bernoulli polynomials of the second kind of order $r$ (see [21]). When $r=1, b_{n}(x)=b_{n}^{(1)}(x),(n \geq 0)$, are called the Bernoulli polynomials of the second kind.

For $n \geq 0$, the falling factorial sequence is defined by

$$
(x)_{0}=1, \quad(x)_{n}=x(x-1) \cdots(x-n+1), \quad(n \geq 1),(\text { see }[21]) .
$$

The Stirling numbers of the first kind are given by

$$
(x)_{n}=\sum_{l=0}^{n} S_{1}(n, l) x^{l} \quad(n \geq 0),(\text { see }[21]) .
$$

As an inversion formula of (12), the Stirling numbers of the second kind are defined as

$$
x^{n}=\sum_{l=0}^{n} S_{2}(n, l)(x)_{l} \quad(n \geq 0),(\text { see }[1,16,21]) .
$$

In [9], the degenerate Stirling numbers of the first kind are defined by Kim and Kim as follows:

$$
(x)_{n}=\sum_{l=0}^{n} S_{1, \lambda}(n, l)(x)_{l, \lambda} \quad(n \geq 0) .
$$


Note that $\lim _{\lambda \rightarrow 0} S_{1, \lambda}(n, l)=S_{1}(n, l),(n \geq 0)$. As an inversion formula of (14), the Stirling numbers of the second kind are given by

$$
(x)_{n, \lambda}=\sum_{l=0}^{n} S_{2, \lambda}(n, l)(x)_{l} \quad(n \geq 0),(\text { see }[11]) .
$$

Thus, by (14) and (15), we get

$$
\frac{1}{k !}\left(\log _{\lambda}(1+t)\right)^{k}=\sum_{n=k}^{\infty} S_{1, \lambda}(n, k) \frac{t^{n}}{n !} \quad(k \geq 0),(\text { see [9] })
$$

and

$$
\frac{1}{k !}\left(e_{\lambda}(t)-1\right)^{k}=\sum_{n=k}^{\infty} S_{2, \lambda}(n, k) \frac{t^{n}}{n !} \quad(k \geq 0),(\text { see }[11]) .
$$

In [16], the degenerate Bell polynomials are defined by

$$
e^{x\left(e_{\lambda}(t)-1\right)}=\sum_{n=0}^{\infty} \operatorname{Bel}_{n, \lambda}(x) \frac{t^{n}}{n !}
$$

When $x=1, \operatorname{Bel}_{n, \lambda}=\operatorname{Bel}_{n, \lambda}(1)$ are called the degenerate Bell numbers.

From (18), we note that

$$
\operatorname{Bel}_{n, \lambda}(x+y)=\sum_{l=0}^{n}\left(\begin{array}{l}
n \\
l
\end{array}\right) \operatorname{Bel}_{l, \lambda}(x) \operatorname{Bel}_{n-l, \lambda}(y), \quad(n \geq 0) .
$$

For the rest of this section, we will briefly go over some basic facts about umbral calculus. For the details on this fascinating mathematics, the interested reader may refer to [6, 21, $22,26]$. Let $\mathbb{C}$ be the field of complex numbers, and let $\mathcal{F}$ be the set of all formal power series in the variable $t$ over $\mathbb{C}$, given by

$$
\mathcal{F}=\left\{f(t)=\sum_{k=0}^{\infty} a_{k} \frac{t^{k}}{k !} \mid a_{k} \in \mathbb{C}\right\}
$$

Let $\mathbb{P}=\mathbb{C}[x]$, and let $\mathbb{P}^{*}$ be the vector space of all linear functionals on $\mathbb{P}$. The action of the linear functional $L \in \mathbb{P}^{*}$ on a polynomial $p(x)$ is denoted by $\langle L \mid p(x)\rangle$, which is linearly extended by the rule

$$
\left\langle c L+c^{\prime} L^{\prime} \mid p(x)\right\rangle=c\langle L \mid p(x)\rangle+c^{\prime}\left\langle L^{\prime} \mid p(x)\right\rangle
$$

where $c, c^{\prime} \in \mathbb{C}$.

For $f(t)=\sum_{k=0}^{\infty} a_{k} \frac{t^{k}}{k !}$, the action $\langle f(t) \mid \cdot\rangle$ of the linear functional $f(t)$ on $\mathbb{P}$ is defined by

$$
\left\langle f(t) \mid x^{n}\right\rangle=a_{n} \quad \text { for all } n \geq 0(\text { see }[1,19,21,22])
$$

Thus, by (20), we get

$$
\left\langle t^{k} \mid x^{n}\right\rangle=n ! \delta_{n, k} \quad(n, k \geq 0),(\text { see }[1,10,19,21]),
$$


where $\delta_{n, k}$ is the Kronecker symbol.

Let

$$
f_{L}(t)=\sum_{k=0}^{\infty}\left\langle L \mid x^{k}\right\rangle \frac{t^{k}}{k !}
$$

Then, by (21), we have

$$
\left\langle f_{L}(t) \mid x^{n}\right\rangle=\sum_{k=0}^{\infty} \frac{\left\langle L \mid x^{k}\right\rangle}{k !}\left\langle t^{k} \mid x^{n}\right\rangle=\left\langle L \mid x^{n}\right\rangle
$$

Moreover, the map $L \mapsto f_{L}(t)$ is a vector space isomorphism from $\mathbb{P}^{*}$ onto $\mathcal{F}$.

Henceforth, $\mathcal{F}$ denotes both the algebra of the formal power series in $t$ and the vector space of all linear functionals on $\mathbb{P}$, and so an element of $\mathcal{F}$ will be thought of as both a formal power series and a linear functional. We call $\mathcal{F}$ the umbral algebra. The umbral calculus is the study of the umbral algebra. Note that $\left\langle e^{y t} \mid p(x)\right\rangle=p(y)$. The order $O(f(t))$ of a power series $f(t)(\neq 0)$ is the smallest integer $k$ for which the coefficient of $t^{k}$ does not vanish. An invertible series is a series $f(t)$ with $O(f(t))=0$, while a delta series is a series $f(t)$ with $O(f(t))=1$. Let $f(t), g(t) \in \mathcal{F}$ with $O(f(t))=1$ and $O(g(t))=0$. Then it is known that there exists a unique sequence $s_{n}(x),(n \geq 0)$, of polynomials satisfying the conditions

$$
\left\langle g(t)(f(t))^{k} \mid s_{n}(x)\right\rangle=n ! \delta_{n, k} \quad(n, k \geq 0),(\text { see }[21, \text { p. 17] }) .
$$

The sequence $s_{n}(x)$ is called the Sheffer sequence for $(g(t), f(t))$, which we denote by $s_{n}(x) \sim$ $(g(t), f(t))$. For $p_{n}(x) \sim(1, f(t)), q_{n}(x) \sim(1, g(t))$, we have the transfer formula

$$
q_{n}(x)=x\left(\frac{f(t)}{g(t)}\right)^{n} x^{-1} p_{n}(x), \quad(n \geq 1),(\text { see }[21, \text { p. } 51]) .
$$

It is well known that $s_{n}(x) \sim(g(t), f(t))$ if and only if

$$
\frac{1}{g(\bar{f}(t))} e^{x \bar{f}(t)}=\sum_{k=0}^{\infty} \frac{s_{k}(x)}{k !} t^{k} \quad(\text { see [21, p. 107]) }
$$

for all $x \in \mathbb{C}$, where $\bar{f}(t)$ is the compositional inverse of $f(t)$ satisfying $f(\bar{f}(t))=\bar{f}(f(t))=t$.

Let $s_{n}(x) \sim(g(t), f(t))$, and let $r_{n}(x) \sim(h(t), l(t))$. Then we have

$$
s_{n}(x)=\sum_{m=0}^{\infty} a_{n, m} r_{m}(x), \quad(n \geq 0),
$$

where

$$
a_{n, m}=\frac{1}{m !}\left\langle\frac { h ( \overline { f } ( t ) ) } { g ( \overline { f } ( t ) ) } \left( l(\bar{f}(t))^{m}\left|x^{n}\right\rangle \quad\right.\right. \text { (see [21, p. 132]). }
$$




\section{Some identities of degenerate Bell polynomials}

From (18), we note that

$$
\begin{aligned}
\sum_{n=0}^{\infty} \operatorname{Bel}_{n, \lambda}(x) \frac{t^{n}}{n !} & =e^{x\left(e_{\lambda}(t)-1\right)}=\sum_{l=0}^{\infty} x^{l} \sum_{n=l}^{\infty} S_{2, \lambda}(n, l) \frac{t^{n}}{n !} \\
& =\sum_{n=0}^{\infty}\left(\sum_{l=0}^{n} S_{2, \lambda}(n, l) x^{l}\right) \frac{t^{n}}{n !} .
\end{aligned}
$$

Thus, by (27), we get

$$
\operatorname{Bel}_{n, \lambda}(x)=\sum_{l=0}^{n} S_{2, \lambda}(n, l) x^{l} \quad(n \geq 0) .
$$

On the other hand, by (18), we have

$$
\begin{aligned}
e^{x\left(e_{\lambda}(t)-1\right)} & =e^{-x} e^{x\left(e_{\lambda}(t)\right)}=\frac{1}{e^{x}} \sum_{l=0}^{\infty} \frac{x^{l}}{l !} e_{\lambda}^{l}(t)=e^{-x} \sum_{l=0}^{\infty} \frac{x^{l}}{l !} \sum_{n=0}^{\infty}(l)_{n, \lambda} \frac{t^{n}}{n !} \\
& =\sum_{n=0}^{\infty}\left\{\frac{1}{e^{x}} \sum_{l=0}^{\infty} \frac{x^{l}}{l !}(l)_{n, \lambda}\right\} \frac{t^{n}}{n !} .
\end{aligned}
$$

Now, (18) and (29) together yield the following Dobinski-type formula.

Lemma 1 For $n \geq 0$, we have

$$
\operatorname{Bel}_{n, \lambda}(x)=\frac{1}{e^{x}} \sum_{l=0}^{\infty} \frac{(l)_{n, \lambda}}{l !} x^{l} .
$$

For $n \in \mathbb{N}$, with the help of Lemma 1 , we obtain

$$
\begin{aligned}
& x \sum_{k=1}^{n}\left(\begin{array}{c}
n-1 \\
k-1
\end{array}\right) \operatorname{Bel}_{k-1, \lambda}(x)(1-\lambda)_{n-k, \lambda} \\
& \quad=x \sum_{k=1}^{n}\left(\begin{array}{l}
n-1 \\
k-1
\end{array}\right)(1-\lambda)_{n-k, \lambda} e^{-x} \sum_{l=0}^{\infty} \frac{(l)_{k-1, \lambda}}{l !} x^{l} \\
& \quad=x e^{-x} \sum_{l=0}^{\infty} \frac{x^{l}}{l !} \sum_{k=0}^{n-1}\left(\begin{array}{c}
n-1 \\
k
\end{array}\right)(1-\lambda)_{n-1-k, \lambda}(l)_{k, \lambda} \\
& =x e^{-x} \sum_{l=0}^{\infty} \frac{x^{l}}{l !}(l+1-\lambda)_{n-1, \lambda}=x e^{-x} \sum_{l=0}^{\infty} \frac{x^{l}}{(l+1) !}(l+1)_{n, \lambda} \\
& =e^{-x} \sum_{l=1}^{\infty} \frac{x^{l}}{l !}(l)_{n, \lambda}=\operatorname{Bel}_{n, \lambda}(x) .
\end{aligned}
$$

Therefore, by (30), we have shown the following proposition. 
Proposition 2 For $n \in \mathbb{N}$, we have

$$
\operatorname{Bel}_{n, \lambda}(x)=x \sum_{k=1}^{n}\left(\begin{array}{l}
n-1 \\
k-1
\end{array}\right) \operatorname{Bel}_{k-1, \lambda}(x)(1-\lambda)_{n-k, \lambda}
$$

By Proposition 2, we get

$$
\begin{aligned}
\frac{\operatorname{Bel}_{n, \lambda}(x)}{x} & =\sum_{k=1}^{n}\left(\begin{array}{c}
n-1 \\
k-1
\end{array}\right) \operatorname{Bel}_{k-1, \lambda}(x)(1-\lambda)_{n-k, \lambda} \\
& =\sum_{k=0}^{n-1}\left(\begin{array}{c}
n-1 \\
k
\end{array}\right) \operatorname{Bel}_{k, \lambda}(x)(1-\lambda)_{n-k-1, \lambda} \\
& =\sum_{k=0}^{n-1}\left(\begin{array}{c}
n-1 \\
k
\end{array}\right) \operatorname{Bel}_{k, \lambda}(x)(1)_{n-k, \lambda} \quad(n \in \mathbb{N}) .
\end{aligned}
$$

By using (31), we may proceed to deriving the following equation:

$$
\begin{aligned}
\frac{\operatorname{Bel}_{n+1, \lambda}(x)}{x} & =\sum_{k=0}^{n}\left(\begin{array}{l}
n \\
k
\end{array}\right) \operatorname{Bel}_{k, \lambda}(x)(1)_{n+1-k, \lambda} \\
& =\sum_{k=0}^{n}\left(\begin{array}{l}
n \\
k
\end{array}\right) \operatorname{Bel}_{k, \lambda}(x)(1)_{n-k, \lambda}(1-(n-k) \lambda) \\
& =\sum_{k=0}^{n}\left(\begin{array}{l}
n \\
k
\end{array}\right) \operatorname{Bel}_{k, \lambda}(x)(1)_{n-k, \lambda}-\lambda \sum_{k=0}^{n-1}\left(\begin{array}{l}
n \\
k
\end{array}\right)(n-k) \operatorname{Bel}_{k, \lambda}(x)(1)_{n-k, \lambda} \\
& =\sum_{k=0}^{n}\left(\begin{array}{l}
n \\
k
\end{array}\right) \operatorname{Bel}_{k, \lambda}(x)(1)_{n-k, \lambda}-n \lambda \sum_{k=0}^{n-1}\left(\begin{array}{c}
n-1 \\
k
\end{array}\right) \operatorname{Bel}_{k, \lambda}(x)(1)_{n-k, \lambda} \\
& =\sum_{k=0}^{n}\left(\begin{array}{l}
n \\
k
\end{array}\right) \operatorname{Bel}_{k, \lambda}(x)(1)_{n-k, \lambda}-n \lambda \frac{\operatorname{Bel}_{n, \lambda}(x)}{x} \quad(n \in \mathbb{N}) .
\end{aligned}
$$

By (32), we get

$$
\sum_{k=0}^{n}\left(\begin{array}{l}
n \\
k
\end{array}\right) \operatorname{Bel}_{k, \lambda}(x)(1)_{n-k, \lambda}=\frac{\operatorname{Bel}_{n+1, \lambda}(x)}{x}+n \lambda \frac{\operatorname{Bel}_{n, \lambda}(x)}{x} \quad(n \in \mathbb{N}) .
$$

From (33), we note that

$$
\begin{aligned}
\sum_{n=0}^{\infty} \frac{d}{d x} \operatorname{Bel}_{n, \lambda}(x) \frac{t^{n}}{n !} & =\frac{d}{d x} e^{x\left(e_{\lambda}(t)-1\right)}=\left(e_{\lambda}(t)-1\right) e^{x\left(e_{\lambda}(t)-1\right)} \\
& =e_{\lambda}(t) e^{x\left(e_{\lambda}(t)-1\right)}-e^{x\left(e_{\lambda}(t)-1\right)} \\
& =\sum_{n=0}^{\infty}\left(\sum_{m=0}^{n}\left(\begin{array}{c}
n \\
m
\end{array}\right) \operatorname{Bel}_{m, \lambda}(x)(1)_{n-m, \lambda}\right) \frac{t^{n}}{n !}-\sum_{n=0}^{\infty} \operatorname{Bel}_{n, \lambda}(x) \frac{t^{n}}{n !} \\
& =\sum_{n=0}^{\infty}\left\{\frac{\operatorname{Bel}_{n+1, \lambda}(x)}{x}+n \lambda \frac{\operatorname{Bel}_{n, \lambda}(x)}{x}-\operatorname{Bel}_{n, \lambda}(x)\right\} \frac{t^{n}}{n !} .
\end{aligned}
$$


By comparing the coefficients on both sides of (34), we obtain the following theorem.

Theorem 3 For $n \geq 1$, we have

$$
\frac{d}{d x} \operatorname{Bel}_{n, \lambda}(x)=\frac{1}{x}\left\{\operatorname{Bel}_{n+1, \lambda}(x)+(n \lambda-x) \operatorname{Bel}_{n, \lambda}(x)\right\} .
$$

Let

$$
\mathbb{P}_{n}=\{p(x) \in \mathbb{C}[x] \mid \operatorname{deg} p(x) \leq n\}, \quad(n \geq 0)
$$

For $p(x) \in \mathbb{P}_{n}$, let

$$
p(x)=\sum_{m=0}^{n} a_{m} \operatorname{Bel}_{m, \lambda}(x), \quad(n \geq 0)
$$

Then, by (22) and noting that $\operatorname{Bel}_{n, \lambda}(x) \sim\left(1, \log _{\lambda}(1+t)\right)$, we get

$$
\left\langle\left(\log _{\lambda}(1+t)\right)^{m} \mid p(x)\right\rangle=\sum_{l=0}^{n} a_{l}\left\langle\left(\log _{\lambda}(1+t)\right)^{m} \mid \operatorname{Bel}_{l, \lambda}(x)\right\rangle=\sum_{l=0}^{n} a_{l} \delta_{l, m} l !=a_{m} m !
$$

Hence, by (36), we get

$$
a_{m}=\frac{1}{m !}\left\langle\left(\log _{\lambda}(1+t)\right)^{m} \mid p(x)\right\rangle, \quad(m \geq 0) .
$$

Therefore, by (35) and (37), we obtain the following lemma.

Lemma 4 For $p(x) \in \mathbb{P}_{n}$, we have

$$
p(x)=\sum_{m=0}^{n} a_{m} \operatorname{Bel}_{m, \lambda}(x)
$$

where

$$
a_{m}=\frac{1}{m !}\left\langle\left(\log _{\lambda}(1+t)\right)^{m} \mid p(x)\right\rangle
$$

Let us take $p(x)=E_{n}(x) \in \mathbb{P}_{n}$. Then, by (8) and Lemma 4, we get

$$
E_{n}(x)=\sum_{m=0}^{n} a_{m} \operatorname{Bel}_{m, \lambda}(x), \quad(n \geq 0)
$$

where

$$
\begin{aligned}
a_{m} & =\frac{1}{m !}\left\langle\left(\log _{\lambda}(1+t)\right)^{m} \mid E_{n}(x)\right\rangle \\
& =\frac{1}{m !} \sum_{l=m}^{n}\left(\begin{array}{l}
n \\
l
\end{array}\right) E_{n-l}\left\langle\left(\log _{\lambda}(1+t)\right)^{m} \mid x^{l}\right\rangle
\end{aligned}
$$




$$
\begin{aligned}
& =\sum_{l=m}^{n}\left(\begin{array}{l}
n \\
l
\end{array}\right) E_{n-l}\left\langle\sum_{k=m}^{l} S_{1, \lambda}(k, m) \frac{t^{k}}{k !} \mid x^{l}\right\rangle \\
& =\sum_{l=m}^{n}\left(\begin{array}{l}
n \\
l
\end{array}\right) E_{n-l} \sum_{k=m}^{l} S_{1, \lambda}(k, m) \frac{1}{k !}\left\langle t^{k} \mid x^{l}\right\rangle \\
& =\sum_{l=m}^{n}\left(\begin{array}{l}
n \\
l
\end{array}\right) E_{n-l} S_{1, \lambda}(l, m) .
\end{aligned}
$$

Thus, (38) and (39) together give the following theorem.

Theorem 5 For $n \geq 0$, we have

$$
E_{n}(x)=\sum_{m=0}^{n}\left\{\sum_{l=m}^{n}\left(\begin{array}{l}
n \\
l
\end{array}\right) E_{n-l} S_{1, \lambda}(l, m)\right\} \operatorname{Bel}_{m, \lambda}(x) .
$$

Now, in order to get the inversion formula of (28), we take $p(x)=x^{n} \in \mathbb{P}_{n}$. Then, by Lemma 4, we get

$$
x^{n}=\sum_{k=0}^{n} a_{k} \operatorname{Bel}_{k, \lambda}(x)
$$

where

$$
\begin{aligned}
a_{k} & =\frac{1}{k !}\left\langle\left(\log _{\lambda}(1+t)\right)^{k} \mid x^{n}\right\rangle=\sum_{m=k}^{n} \frac{S_{1, \lambda}(m, k)}{m !}\left\langle t^{m} \mid x^{n}\right\rangle \\
& =\sum_{m=k}^{n} \frac{S_{1, \lambda}(m, k)}{m !} n ! \delta_{m, n}=S_{1, \lambda}(n, k) .
\end{aligned}
$$

Now, from (40) and (41), we have the following theorem.

\section{Theorem 6 For $n \geq 0$, we have}

$$
x^{n}=\sum_{k=0}^{n} S_{1, \lambda}(n, k) \operatorname{Bel}_{k, \lambda}(x) .
$$

For $\operatorname{Bel}_{n, \lambda}(x) \sim\left(1, \log _{\lambda}(1+t)\right), x^{n} \sim(1, t)$, by using the transfer formula in (23), we get

$$
\begin{aligned}
\operatorname{Bel}_{n, \lambda}(x) & =x\left(\frac{t}{\log _{\lambda}(1+t)}\right)^{n} x^{-1} x^{n} \\
& =x\left(\frac{t}{\log _{\lambda}(1+t)}\right)^{n} \sum_{l=0}^{n-1} S_{2}(n-1, l)(x)_{l} \\
& =x \sum_{l=0}^{n-1} S_{2}(n-1, l)\left(\frac{t}{\log _{\lambda}(1+t)}\right)^{n}(x)_{l} .
\end{aligned}
$$


Now, we observe that

$$
\begin{aligned}
\left(\frac{t}{\log _{\lambda}(1+t)}\right)^{n}(x)_{l} & =\sum_{k=0}^{\infty} b_{k, \lambda}^{(n)} \frac{t^{k}}{k !} \sum_{m=0}^{l} S_{1}(l, m) x^{m} \\
& =\sum_{m=0}^{l} \sum_{k=0}^{m} b_{k, \lambda}^{(n)}\left(\begin{array}{c}
m \\
k
\end{array}\right) x^{m-k} S_{1}(l, m) \\
& =\sum_{m=0}^{l} \sum_{k=0}^{m}\left(\begin{array}{c}
m \\
k
\end{array}\right) S_{1}(l, m) b_{m-k, \lambda}^{(n)} x^{k}
\end{aligned}
$$

Thus, by (42) and (43), we get

$$
\begin{aligned}
\operatorname{Bel}_{n, \lambda}(x) & =x \sum_{l=0}^{n-1} \sum_{m=0}^{l} \sum_{k=0}^{m}\left(\begin{array}{l}
m \\
k
\end{array}\right) S_{1}(l, m) b_{m-k, \lambda}^{(n)} S_{2}(n-1, l) x^{k} \\
& =x \sum_{l=0}^{n-1} \sum_{k=0}^{l} \sum_{m=k}^{l}\left(\begin{array}{l}
m \\
k
\end{array}\right) S_{1}(l, m) b_{m-k, \lambda}^{(n)} S_{2}(n-1, l) x^{k} \\
& =x \sum_{k=0}^{n-1} \sum_{l=k}^{n-1} \sum_{m=k}^{l}\left(\begin{array}{l}
m \\
k
\end{array}\right) S_{1}(l, m) S_{2}(n-1, l) b_{m-k, \lambda}^{(n)} x^{k} .
\end{aligned}
$$

From Proposition 2 and (44), we note that

$$
\begin{aligned}
\frac{\operatorname{Bel}_{n, \lambda}(x)}{x} & =\sum_{k=0}^{n-1} \sum_{l=k}^{n-1} \sum_{m=k}^{l}\left(\begin{array}{l}
m \\
k
\end{array}\right) S_{1}(l, m) S_{2}(n-1, l) b_{m-k, \lambda}^{(n)} x^{k} \\
& =\sum_{k=1}^{n}\left(\begin{array}{l}
n-1 \\
k-1
\end{array}\right) \operatorname{Bel}_{k-1, \lambda}(x)(1-\lambda)_{n-k, \lambda} \quad(n \in \mathbb{N}) .
\end{aligned}
$$

Therefore, by combining (44) with (45), we obtain the following theorem.

Theorem 7 For $n \in \mathbb{N}$, we have

$$
\sum_{k=1}^{n}\left(\begin{array}{c}
n-1 \\
k-1
\end{array}\right) \operatorname{Bel}_{k-1, \lambda}(x)(1-\lambda)_{n-k, \lambda}=\sum_{k=0}^{n-1} \sum_{l=k}^{n-1} \sum_{m=k}^{l}\left(\begin{array}{c}
m \\
k
\end{array}\right) S_{1}(l, m) S_{2}(n-1, l) b_{m-k, \lambda}^{(n)} x^{k}
$$

Let us consider the following two Sheffer sequences:

$$
\beta_{n, \lambda}^{(k)}(x) \sim\left(g(t), f(t)=\frac{1}{\lambda}\left(e^{\lambda t}-1\right)\right), \quad \operatorname{Bel}_{n, \lambda}(x) \sim\left(1, \log _{\lambda}(1+t)\right)
$$

where $g(t)$ is uniquely determined by $\frac{1}{g(\bar{f}(t))}=\frac{\operatorname{Li}_{k, \lambda}\left(1-e_{\lambda}(-t)\right)}{e_{\lambda}(t)-1}$. From (25) and (26), we have

$$
\beta_{n, \lambda}^{(k)}(x)=\sum_{m=0}^{n} a_{n, m} \operatorname{Bel}_{m, \lambda}(x)
$$


where we have

$$
\begin{aligned}
a_{n, m} & =\frac{1}{m !}\left\langle\frac{\operatorname{Li}_{k, \lambda}\left(1-e_{\lambda}(-t)\right)}{e_{\lambda}(t)-1}\left(\log _{\lambda}\left(1+\frac{1}{\lambda} \log (1+\lambda t)\right)\right)^{m} \mid x^{n}\right\rangle \\
& =\sum_{l=m}^{n} \frac{S_{1, \lambda}(l, m)}{l !}\left\langle\frac{\operatorname{Li}_{k, \lambda}\left(1-e_{\lambda}(-t)\right)}{e_{\lambda}(t)-1}\left(\frac{1}{\lambda} \log (1+\lambda t)\right)^{l} \mid x^{n}\right\rangle \\
& =\sum_{l=m}^{n} \frac{S_{1, \lambda}(l, m)}{\lambda^{l}}\left\langle\frac{\operatorname{Li}_{k, \lambda}\left(1-e_{\lambda}(-t)\right)}{e_{\lambda}(t)-1} \frac{1}{l !}(\log (1+\lambda t))^{l} \mid x^{n}\right\rangle \\
& =\sum_{l=m}^{n} \frac{S_{1, \lambda}(l, m)}{\lambda^{l}} \sum_{r=l}^{n} S_{1, \lambda}(r, l) \frac{\lambda^{r}}{r !}\left\langle\frac{\operatorname{Li}_{k, \lambda}\left(1-e_{\lambda}(-t)\right)}{e_{\lambda}(t)-1} t^{r} \mid x^{n}\right\rangle \\
& =\sum_{l=m}^{n} \sum_{r=l}^{n}\left(\begin{array}{l}
n \\
r
\end{array}\right) S_{1, \lambda}(l, m) S_{1, \lambda}(r, l) \lambda^{r-l}\left\langle\frac{\operatorname{Li}_{k, \lambda}\left(1-e_{\lambda}(-t)\right)}{e_{\lambda}(t)-1} \mid x^{n-r}\right\rangle \\
& =\sum_{l=m}^{n} \sum_{r=l}^{n}\left(\begin{array}{l}
n \\
r
\end{array}\right) S_{1, \lambda}(l, m) S_{1, \lambda}(r, l) \lambda^{r-l} \sum_{j=0}^{\infty} \beta_{j, \lambda}^{(k)} \frac{1}{j !}\left\langle t^{j} \mid x^{n-r}\right\rangle \\
& =\sum_{l=m}^{n} \sum_{r=l}^{n}\left(\begin{array}{l}
n \\
r
\end{array}\right) S_{1, \lambda}(l, m) S_{1, \lambda}(r, l) \lambda^{r-l} \sum_{j=0}^{n-r} \beta_{j, \lambda}^{(k)} \frac{1}{j !}(n-r) ! \delta_{j, n-r} \\
& =\sum_{l=m}^{n} \sum_{r=l}^{n}\left(\begin{array}{l}
n \\
r
\end{array}\right) S_{1, \lambda}(l, m) S_{1, \lambda}(r, l) \lambda^{r-l} \beta_{n-r, \lambda}^{(k)} .
\end{aligned}
$$

Therefore, by (46) and (47), we obtain the following theorem.

Theorem 8 For $n \geq 0$, we have

$$
\beta_{n, \lambda}^{(k)}(x)=\sum_{m=0}^{n}\left\{\sum_{l=m}^{n} \sum_{r=l}^{n}\left(\begin{array}{l}
n \\
r
\end{array}\right) S_{1, \lambda}(l, m) S_{1, \lambda}(r, l) \lambda^{r-l} \beta_{n-r, \lambda}^{(k)}\right\} \operatorname{Bel}_{m, \lambda}(x) .
$$

For $\operatorname{Bel}_{n, \lambda}(x) \sim\left(1, \log _{\lambda}(1+t)\right),(x)_{n} \sim\left(1, e^{t}-1\right)$, we have

$$
\operatorname{Bel}_{n, \lambda}(x)=\sum_{m=0}^{n} a_{n, m}(x)_{m}
$$

where we have

$$
\begin{aligned}
a_{n, m} & =\frac{1}{m !}\left\langle\left(e^{\left(e_{\lambda}(t)-1\right)}-1\right)^{m} \mid x^{n}\right\rangle \\
& =\frac{1}{m !} \sum_{l=0}^{m}\left(\begin{array}{c}
m \\
l
\end{array}\right)(-1)^{m-l}\left\langle\left(e^{l\left(e_{\lambda}(t)-1\right)}\right) \mid x^{n}\right\rangle \\
& =\frac{1}{m !} \sum_{l=0}^{m}\left(\begin{array}{c}
m \\
l
\end{array}\right)(-1)^{m-l} \sum_{k=0}^{n} \operatorname{Bel}_{k, \lambda}(l) \frac{1}{k !}\left\langle t^{k} \mid x^{n}\right\rangle \\
& =\frac{1}{m !} \sum_{l=0}^{m}\left(\begin{array}{c}
m \\
l
\end{array}\right)(-1)^{m-l} \operatorname{Bel}_{n, \lambda}(l) .
\end{aligned}
$$

Now, by (48) and (49), we get the following theorem. 
Theorem 9 For $n \geq 0$, we have

$$
\operatorname{Bel}_{n, \lambda}(x)=\sum_{m=0}^{n}\left\{\frac{1}{m !} \sum_{l=0}^{m}\left(\begin{array}{c}
m \\
l
\end{array}\right)(-1)^{m-l} \operatorname{Bel}_{n, \lambda}(l)\right\}(x)_{m} .
$$

Consider the following two Sheffer sequences:

$$
\operatorname{Bel}_{n, \lambda}(x) \sim\left(1, \log _{\lambda}(1+t)\right), \quad b_{n}(x) \sim\left(\frac{t}{e^{t}-1}, e^{t}-1\right) .
$$

Then, by (25) and (26), we get

$$
\operatorname{Bel}_{n, \lambda}(x)=\sum_{m=0}^{n} a_{n, m} b_{m}(x)
$$

where we have

$$
\begin{aligned}
a_{n, 0} & =\left\langle\frac{e_{\lambda}(t)-1}{e^{e_{\lambda}(t)-1}-1} \mid x^{n}\right\rangle=\left\langle\sum_{l=0}^{\infty} \frac{B_{l}}{l !}\left(e_{\lambda}(t)-1\right)^{l} \mid x^{n}\right\rangle \\
& =\sum_{m=0}^{\infty} \sum_{l=0}^{m} \frac{B_{l}}{m !} S_{2, \lambda}(m, l)\left\langle t^{m} \mid x^{n}\right\rangle \\
& =\sum_{m=0}^{n} \sum_{l=0}^{m} \frac{B_{l}}{m !} S_{2, \lambda}(m, l) n ! \delta_{n, m}=\sum_{l=0}^{n} S_{2, \lambda}(n, l) B_{l},
\end{aligned}
$$

and, for $m \geq 1$,

$$
\begin{aligned}
a_{n, m} & =\frac{1}{m !}\left\langle\frac{e_{\lambda}(t)-1}{e^{e_{\lambda}(t)-1}-1}\left(e^{e_{\lambda}(t)-1}-1\right)^{m} \mid x^{n}\right\rangle \\
& =\frac{1}{m !}\left\langle\left(e^{e_{\lambda}(t)-1}-1\right)^{m-1} e_{\lambda}(t) \mid x^{n}\right\rangle-\frac{1}{m !}\left\langle\left(e^{e_{\lambda}(t)-1}-1\right)^{m-1} \mid x^{n}\right\rangle \\
& =\frac{1}{m !} \sum_{k=0}^{n} \frac{(1)_{k, \lambda}}{k !}\left\langle\left(e^{e_{\lambda}(t)-1}-1\right)^{m-1} t^{k} \mid x^{n}\right\rangle-\frac{1}{m !}\left\langle\left(e^{e_{\lambda}(t)-1}-1\right)^{m-1} \mid x^{n}\right\rangle \\
& \left.=\frac{1}{m !} \sum_{k=0}^{n}\left(\begin{array}{l}
n \\
k
\end{array}\right)(1)_{k, \lambda}\left|\left(e^{e_{\lambda}(t)-1}-1\right)^{m-1}\right| x^{n-k}\right\rangle-\frac{1}{m !}\left\langle\left(e^{e_{\lambda}(t)-1}-1\right)^{m-1} \mid x^{n}\right\rangle \\
& =\frac{1}{m !} \sum_{k=0}^{n-1}\left(\begin{array}{l}
n \\
k
\end{array}\right)(1)_{n-k, \lambda}\left\langle\left(e^{e_{\lambda}(t)-1}-1\right)^{m-1} \mid x^{k}\right\rangle \\
& =\frac{1}{m !} \sum_{k=0}^{n-1}\left(\begin{array}{l}
n \\
k
\end{array}\right)(1)_{n-k, \lambda} \sum_{l=0}^{m-1}\left(\begin{array}{c}
m-1 \\
l
\end{array}\right)(-1)^{m-1-l}\left\langle e^{l\left(e_{\lambda}(t)-1\right)} \mid x^{k}\right\rangle \\
& =\frac{1}{m !} \sum_{k=0}^{n-1} \sum_{l=0}^{m-1}\left(\begin{array}{l}
n \\
k
\end{array}\right)(1)_{n-k, \lambda}\left(\begin{array}{c}
m-1 \\
l
\end{array}\right)(-1)^{m-1-l} \operatorname{Bel}_{k, \lambda}(l) .
\end{aligned}
$$

Now, (50)-(52) altogether yield the following result. 
Theorem 10 For $n \geq 0$, we have

$$
\begin{aligned}
\operatorname{Bel}_{n, \lambda}(x)= & \sum_{l=0}^{n} S_{2, \lambda}(n, l) B_{l} \\
& +\sum_{m=1}^{n}\left\{\frac{1}{m !} \sum_{k=0}^{n-1} \sum_{l=0}^{m-1}\left(\begin{array}{l}
n \\
k
\end{array}\right)(1)_{n-k, \lambda}\left(\begin{array}{c}
m-1 \\
l
\end{array}\right)(-1)^{m-1-l} \operatorname{Bel}_{k, \lambda}(l)\right\} b_{n}(x) .
\end{aligned}
$$

\section{Conclusion}

We derived Dobinski-type formulas, recurrence relations, and the derivatives for the degenerate Bell polynomials. After finding a formula expressing any polynomial in terms of the degenerate Bell polynomials, we applied this formula to Euler polynomials and powers of $x$. We obtained a relation involving the degenerate Bell polynomials, Stirling numbers of the first and second kinds, and the higher-order degenerate Bernoulli polynomials of the second kind. In addition, by using the general formula of expressing one Sheffer polynomial in terms of another Sheffer polynomial, we expressed Euler polynomials and modified degenerate poly-Bernoulli polynomials in terms of the degenerate Bell polynomials and also represented the degenerate Bell polynomials in terms of degenerate Bernoulli polynomials of the second kind and falling factorials.

As we briefly mentioned in Sect. 1, degenerate versions of many special polynomials and numbers have applications to identities of symmetry, differential equations, and probability theory as well as to number theory and combinatorics. In more detail, some new combinatorial identities were found from infinite families of ordinary differential equations which are satisfied by the generating functions of some degenerate special polynomials (see [12]). Many identities of symmetry have been discovered for various degenerate versions of quite a few special polynomials in recent years (see [13]). The generating functions of the moments of certain random variables were used in order to derive some identities connecting some special numbers and moments of random variables (see [18]). As one of our future projects, we would like to continue to study degenerate versions of certain special polynomials and numbers and their applications to physics, science, and engineering as well as to mathematics.

\section{Acknowledgements}

We would like to thank the referees for their useful comments and suggestions. Also, we thank Friday mathematics seminar team of the Kwangwoon University for suggesting the present research subject.

\section{Funding}

This research received no external funding.

Availability of data and materials

Not applicable.

Competing interests

The authors declare that they have no competing interests.

Authors' contributions

TK and DSK conceived of the framework and structured the whole paper; TK and DSK wrote the paper; LCJ, HYK, and HL checked the results of the paper; DSK and TK completed the revision of the article. All authors have read and agreed to the published version of the manuscript.

\section{Author details}

'Department of Mathematics, Kwangwoon University, Seoul 139-701, Republic of Korea. ${ }^{2}$ Department of Mathematics, Sogang University, Seoul 121-742, Republic of Korea. ${ }^{3}$ Graduate School of Education, Konkuk University, Seoul 143-701, Republic of Korea. 


\section{Publisher's Note}

Springer Nature remains neutral with regard to jurisdictional claims in published maps and institutional affiliations.

Received: 4 August 2020 Accepted: 21 September 2020 Published online: 25 September 2020

\section{References}

1. Araci, S.: Novel identities involving Genocchi numbers and polynomials arising from applications of umbral calculus. Appl. Math. Comput. 233, 599-607 (2014)

2. Bell, E.T.: Exponential numbers. Am. Math. Mon. 41, 411-419 (1934)

3. Carlitz, L.: A degenerate Staudt-Clausen theorem. Arch. Math. (Basel) 7, 28-33 (1956)

4. Carlitz, L.: Degenerate Stirling, Bernoulli and Eulerian numbers. Util. Math. 15, 51-88 (1979)

5. Dere, R., Simsek, Y: Applications of umbral algebra to some special polynomials. Adv. Stud. Contemp. Math. (Kyungshang) 22(3), 433-438 (2012)

6. Dere, R., Simsek, Y., Srivastava, H.M.: A unified presentation of three families of generalized Apostol type polynomials based upon the theory of the umbral calculus and the umbral algebra. J. Number Theory 133, 3245-3263 (2013)

7. El-Desouky, B.S., Mustafa, A.: New results on higher-order Daehee and Bernoulli numbers and polynomials. Adv. Differ. Equ. 2016, 32 (2016)

8. Khan, W.A., Ahmad, M.: Partially degenerate poly-Bernoulli polynomials associated with Hermite polynomials. Adv. Stud. Contemp. Math. (Kyungshang) 28(3), 487-496 (2018)

9. Kim, D.S., Kim, T.: A note on a new type of degenerate Bernoulli numbers. Russ. J. Math. Phys. 27(2), 227-235 (2020)

10. Kim, D.S., Kim, T.: Degenerate Sheffer sequences and $\lambda$-Sheffer sequences. J. Math. Anal. Appl. Available online 19 August 2020, 124521. https://www.sciencedirect.com/science/article/pii/S0022247X20306831

11. Kim, T:: A note on degenerate Stirling polynomials of the second kind. Proc. Jangjeon Math. Soc. 20(3), 319-331 (2017)

12. Kim, T., Kim, D.S.: Identities involving degenerate Euler numbers and polynomials arising from non-linear differential equations. J. Nonlinear Sci. Appl. 9, 2086-2098 (2016)

13. Kim, T., Kim, D.S.: Identities of symmetry for degenerate Euler polynomials and alternating generalized falling factorial sums. Iran. J. Sci. Technol. Trans. A, Sci. 41(4), 939-949 (2017)

14. Kim, T., Kim, D.S.: Degenerate polyexponential functions and degenerate Bell polynomials. J. Math. Anal. Appl. 487(2), $124017(2020)$

15. Kim, T., Kim, D.S.: Note on the degenerate gamma function. Russ. J. Math. Phys. 27(3), 353-359 (2020)

16. Kim, T., Kim, D.S., Dolgy, V.: On partially degenerate Bell numbers and polynomials. Proc. Jangjeon Math. Soc. 20(3), 337-345 (2017)

17. Kim, T., Kim, D.S., Kim, H.-Y., Lee, H., Jang, L.-C.: Degenerate poly-Bernoulli polynomials arising from degenerate polylogarithm. Adv. Differ. Equ. 2020, 444 (2020)

18. Kim, T., Kim, D.S., Kim, H.Y., Kwon, J.: Degenerate Stirling polynomials of the second kind and some applications. Symmetry 11(8), 1046 (2019)

19. Ma, Y., Kim, D. S., Kim, T., Kim, H., Lee, H.: Some identities of Lah-Bell polynomials. Adv. Differ. Equ. 2020510 (2020)

20. Pyo, S.-S.: Degenerate Cauchy numbers and polynomials of the fourth kind. Adv. Stud. Contemp. Math. (Kyungshang) 28(1), 127-138 (2018)

21. Roman, S.: The Umbral Calculus. Pure and Applied Mathematics, vol. 111. Academic Press, Inc. [Harcourt Brace Jovanovich, Publishers], New York (1984)

22. Roman, S., De Land, P., Shiflett, R., Shultz, H.: The umbral calculus and the solution to certain recurrence relations. J. Comb. Inf. Syst. Sci. 8(4), 235-240 (1983)

23. Shang, Y.: Unveiling robustness and heterogeneity through percolation triggered by random-link breakdown. Phys. Rev. E 90, 032820 (2014)

24. Shang, Y.: Effect of link oriented self-healing on resilience of networks. J. Stat. Mech. Theory Exp. 8(8), 083403 (2016)

25. Simsek, Y.: Identities on the Changhee numbers and Apostol-type Daehee polynomials. Adv. Stud. Contemp. Math. (Kyungshang) 27(2), 199-212 (2017)

26. Simsek, Y: Special numbers and polynomials including their generating functions in umbral analysis methods. Axioms 7, 22 (2018). https://doi.org/10.3390/axioms7020022

\section{Submit your manuscript to a SpringerOpen ${ }^{\circ}$ journal and benefit from:}

- Convenient online submission

- Rigorous peer review

- Open access: articles freely available online

- High visibility within the field

- Retaining the copyright to your article

Submit your next manuscript at $\boldsymbol{\nabla}$ springeropen.com 